University of Vermont

UVM ScholarWorks

College of Engineering and Mathematical

College of Engineering and Mathematical

Sciences Faculty Publications

Sciences

5-20-2015

\title{
Text mixing shapes the anatomy of rank-frequency distributions
}

Jake Ryland Williams

University of Vermont

James P. Bagrow

University of Vermont

Christopher M. Danforth

University of Vermont

Peter Sheridan Dodds

University of Vermont

Follow this and additional works at: https://scholarworks.uvm.edu/cemsfac

Part of the Human Ecology Commons, and the Medicine and Health Commons

\section{Recommended Citation}

Williams JR, Bagrow JP, Danforth CM, Dodds PS. Text mixing shapes the anatomy of rank-frequency distributions. Physical Review E. 2015 May 20;91(5):052811.

This Article is brought to you for free and open access by the College of Engineering and Mathematical Sciences at UVM ScholarWorks. It has been accepted for inclusion in College of Engineering and Mathematical Sciences Faculty Publications by an authorized administrator of UVM ScholarWorks. For more information, please contact scholarworks@uvm.edu. 


\title{
Text mixing shapes the anatomy of rank-frequency distributions
}

\author{
Jake Ryland Williams, ${ }^{*}$ James P. Bagrow, ${ }^{\dagger}$ Christopher M. Danforth, ${ }^{\ddagger}$ and Peter Sheridan Dodds ${ }^{\S}$ \\ Department of Mathematics \& Statistics, Vermont Complex Systems Center, Computational Story Lab, \& the Vermont Advanced \\ Computing Core, The University of Vermont, Burlington, Vermont 05401, USA
}

(Received 15 September 2014; revised manuscript received 30 January 2015; published 20 May 2015)

\begin{abstract}
Natural languages are full of rules and exceptions. One of the most famous quantitative rules is Zipf's law, which states that the frequency of occurrence of a word is approximately inversely proportional to its rank. Though this "law" of ranks has been found to hold across disparate texts and forms of data, analyses of increasingly large corpora since the late 1990s have revealed the existence of two scaling regimes. These regimes have thus far been explained by a hypothesis suggesting a separability of languages into core and noncore lexica. Here we present and defend an alternative hypothesis that the two scaling regimes result from the act of aggregating texts. We observe that text mixing leads to an effective decay of word introduction, which we show provides accurate predictions of the location and severity of breaks in scaling. Upon examining large corpora from 10 languages in the Project Gutenberg eBooks collection, we find emphatic empirical support for the universality of our claim.
\end{abstract}

DOI: 10.1103/PhysRevE.91.052811

PACS number(s): 89.75.Da, 89.65.-s, 89.75.Fb

\section{ZIPF'S LAW AND (NON-) UNIVERSALITY}

Given some collection of distinct kinds of objects occurring with frequency $f$ and associated rank $r$ according to decreasing frequency, Zipf's law is said to be fulfilled when ranks and frequencies are approximately inversely proportional:

$$
f(r) \sim r^{-\theta},
$$

typically with $\theta \simeq 1$. Though Zipf's functional form has been found to be a reasonable one for disparate forms of data, ranging from frequencies of words to sizes of cities in Zipf's original work $[1,2]$, its lack of total universality in application to natural languages is now widely acknowledged [3-8].

Recently it was suggested $[3,4]$ that large corpora exhibit two scaling regimes (delineated by some $b>0$ ):

$$
f(r) \sim\left\{\begin{array}{ll}
r^{-\theta}, & : r \leqslant b \\
r^{-\gamma}, & : r>b
\end{array},\right.
$$

the first being that of Zipf $(\theta=1)$ and the second distinctly more variable [4] (though generally $\gamma>1$ ). Ferrer and Solé hypothesized in Ref. [3] that these two regimes reflected a division of natural languages into two lexical subsets-the kernel (core) and unlimited (noncore) lexica.

We observe that in all studies finding dual scalings that the texts analyzed are of mixed origin, that is, they are not derived from a single author or even a single topic. Montemurro indicated in Ref. [4] that combining heterogeneous texts could generate effects that shield investigators from the true underlying nature of this second scaling regime:

To resolve the behavior of those [high rank] words we need a significant increase in volume of data, probably exceeding the length of any conceivable single text. Still, at the same time it is desirable to maintain as high a degree of homogeneity in the texts as possible, in the hope of revealing a more

\footnotetext{
*jake.williams@uvm.edu

†james.bagrow@uvm.edu

${ }^{\ddagger}$ chris.danforth@uvm.edu

§ peter.dodds@uvm.edu
}

complex phenomenology than that simply originating from a bulk average of a wide range of disparate sources.

With this inspiration, we focus on understanding the effects of combining texts of varying heterogeneity-a process we refer to as "text mixing."

\section{STOCHASTIC MODELS}

In the years following Zipf's original work, various stochastic models have been proposed for the generation of natural language vocabularies. The first of these was that proposed by Simon [9] and based on Yule's model of evolution [10]. This work is a powerful companion to understanding Zipf's empirical work and can be seen as the natural antecedent of the rich-gets-richer models $[11,12]$ for growing networks that have interested the complex systems community over recent years. Indeed, perhaps the most important piece we may draw from Simon's model is that a rich-gets-richer mechanism is a reasonable one for the growth of a vocabulary.

An important limitation of Simon's model is that it is only capable of producing a single scaling regime, which, as we know is an incomplete picture. Furthermore, the scalings accessible via the Simon model were strictly less severe than the "universal" $\theta=1$ exponent. So if one assumes the Simon model as truth, with a fixed word introduction rate $\alpha_{0}$, Zipf's exponent should be variable and necessarily less than 1 , though empirically found indistinguishable from 1 , that is, $\theta=1-\alpha_{0}$, with $\alpha_{0} \ll 1$ [9].

Recently, a modification to Simon's model was proposed Gerlach and Altmann in which two types of words could be produced-core and noncore words [5]. As a built-in feature of the core-noncore vocabulary (CNCV) model, the size of the core set of words was prescribed to be finite, while the noncore was allowed to expand indefinitely. Aside from introducing two classes of words, the most important distinction of this model from its predecessor was a rule for the decay in the rate of introduction of new words, $\alpha$. Along with producing the CNCV model they showed that when $\alpha$ decays as a power law with exponent $-\mu$, of the number of unique words, $n$, the relationship between $\mu$ and the lower rank-frequency 
exponent, $\gamma$, is a difference of $\theta$, i.e.,

$$
\alpha(n)=\alpha_{0} n^{-\mu} \Rightarrow f(r) \sim r^{-(\theta+\mu)},
$$

with $\gamma=\theta+\mu$ [5]. The distinction between word types provided a means for postponing the point at which their power law decay would occur, thereby generating two scaling regimes. We note that the severity of the second scaling was only contingent upon the existence of a decay in the rate of introduction of new words and that this decay was imposed rather than the result of the existence of two word types. We are therefore led to find an explicit mechanism capable of producing power-law decaying word introduction rates and hence multiple scaling regimes.

\section{TEXT MIXING}

As we have described, the CNCV model offers a means by which one can obtain a second scaling. The model is, like Simon's, framed as a model of the generation of a vocabulary. However, we are led to question whether lower scalings are a product of vocabulary generation or an artifact of an interaction between disparate texts. Suppose a collection of texts, $\mathcal{C}=$ $\left\{T_{1}, \ldots, T_{k}\right\}$, is read sequentially and that each has rankfrequency distribution of Zipf-Simon form. Upon constructing idealized rank-frequency distributions from empirical data (see Sec. IV), we find that their combined distribution possesses multiple scaling regimes (see Fig. 1). Though each individual vocabulary might have been created without a decay of word introduction, an overlap in the words they use has it seem as though the appearance of new words is rarer by the time the later texts are read. If one reads the texts repeatedly and in permuted orders, the resulting decay in the rate of word introduction likely does not evince itself until the mean text size (mean number of unique words per text) is reached but certainly not before the minimum text size is reached.

Operating under this ansatz-that a text-mixing-derived scaling break, $b$, covaries with the mean number of unique

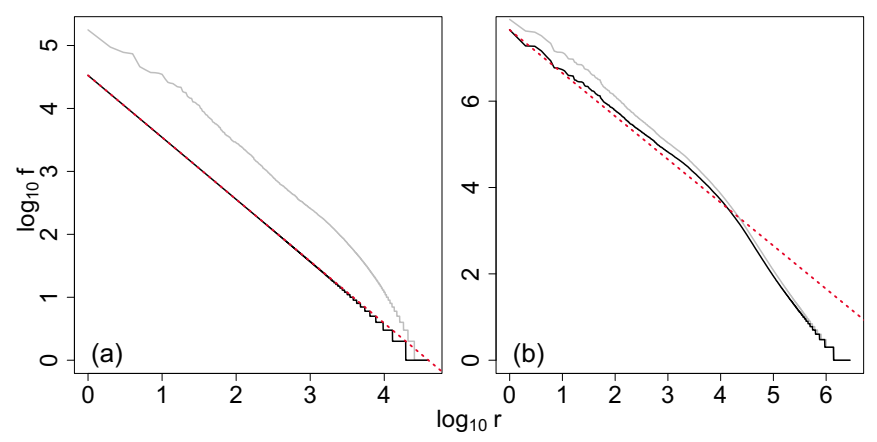

FIG. 1. (Color online) (a) An idealization (black points) of a rank-frequency distribution (gray points) for a single text (The Complete Historical Romances of Georg Ebers) from the English eBooks collection. Idealization is defined by a pure power law of scaling $1-N / M$ where $N$ is the number of unique words and $M$ is the total volume of words and $N / M$ is an estimate for $\alpha$ [red (gray) dashed line, see Sec. IV]. (b) The mixtures of all texts (gray points) and their idealizations (black points) from the English eBooks collection. Note that neither mixture results in a pure power law such as Zipf's [ $\theta=1$, red (gray) dashed line].

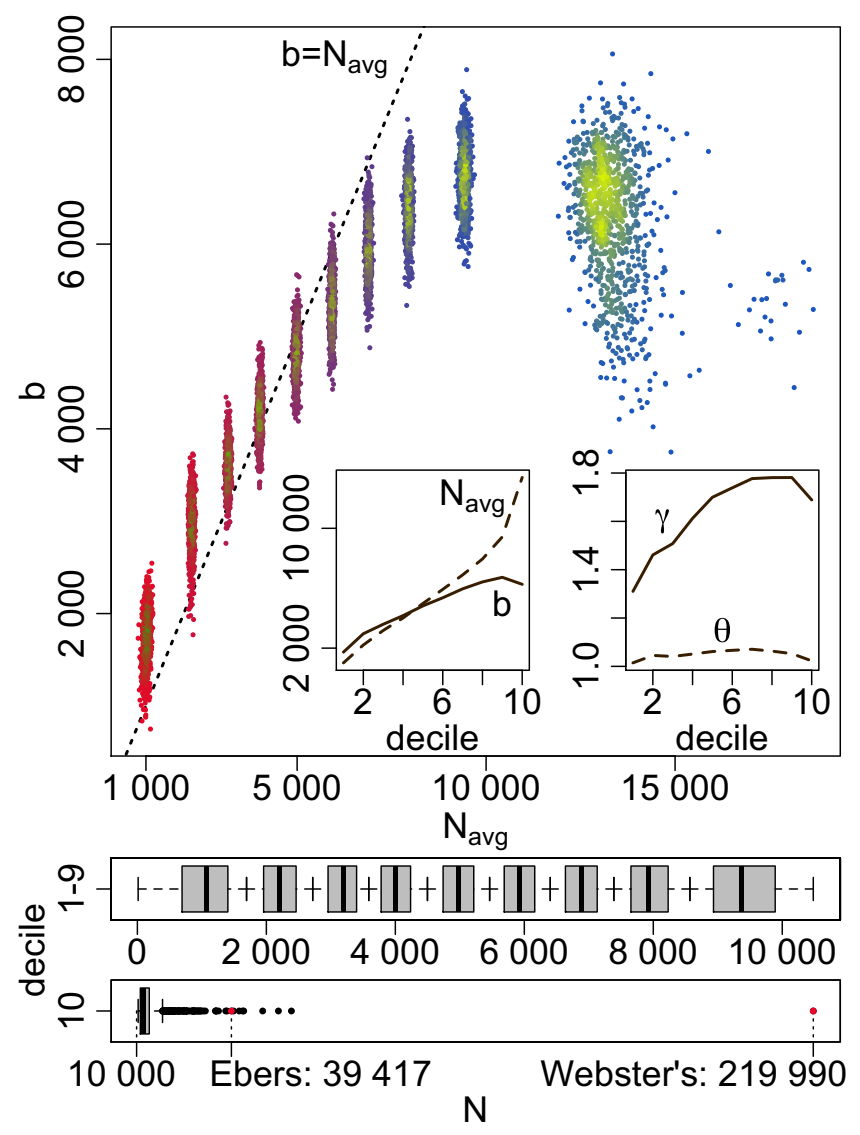

FIG. 2. (Color online) Top: For each of the 10 deciles of the English distribution of text sizes, we measure the parameters $b, \gamma$, $N_{\text {avg }}$, and $\theta$ from 50-book sample corpora. Each cloud represents 1000 sample corpora from deciles 1-10 [low to high, where red to blue (left to right) indicates increasing decile and fading to green or yellow (gray) indicates increasing density]. The line $b=N_{\text {avg }}$ is also presented (dashed line, main axis) and shows that $b$ increases with decile for all but the most extreme $\left(10^{\text {th }}\right)$ decile. Main axes insets show parameter variation across deciles for both $b$ and $N_{\text {avg }}$ (left); $\gamma$ and $\theta$ (right), where we note that Zipf's parameter, $\theta$, is the only one that exhibits signs of stationarity. Bottom: Box plots providing a more detailed look at the 10 deciles of the distribution of text sizes. For clarity we have separated the plots for deciles 1-9 from the $10^{\text {th }}$. This highlights the extreme nature of the later deciles (most notably the $10^{\text {th }}$ ), where the presence of poorly refined texts throw off estimates of $N_{\text {avg }}$, which we also note corresponds to the roll over in the distributions off of the $b=N_{\text {avg }}$ axis above.

words per text, $N_{\text {avg }}$, in a corpus-we investigate thousands of corpora defined by samples from the English eBooks database (see Sec. IV for more details on text sampling and a complete description of the eBooks database). Obtaining 1000 text-sample corpora from each of the 10 deciles of the text-size distribution, we regress for $b$ (see Sec. IV) and record $N_{\text {avg }}$ to find that the two covary strongly along the line $b=N_{\text {avg }}$ for all but the most extreme deciles (see the main axes in Fig. 2, which we return to later in the discussion). We see that this relationship breaks down in the presence of large- $N$ texts, which, upon closer inspection, appear ill formed in the sense of being of mixed origin themselves (e.g., posthumous or longitudinal compendia, dictionaries, encyclopedias, etc.; 
see Sec. IV and Fig. 7 for more details on corpus formation and internally mixed texts). Additionally, we see from these preliminary experiments that both of the quantities, $b$ and $\gamma$, do not appear as universal for a given language (see Fig. 2) but rather depend quite severely on corpus composition. In fact, the only regressed parameter that presents any signs of universality for a language is Zipf's exponent, $\theta$, which remains quite close to 1 . These initial results indicate that hypotheses of the locations of scaling breaks, $b$, corresponding to language-universal lexical-core sizes are in strong need of reevaluation or should be reformulated as corpus relative.

In the following, we run text mixing experiments that measure decay in rates of word introduction directly attributable to mixing texts to predict lower scalings in composite distributions. As we read out texts (in some order) let $m$ be the volume of words observed at any point and $n_{m}$ be the number of distinct words in the volume $m$, which we will refer to as the vocabulary size of the growing text. To exhibit the effects of text mixing we contrast the vocabulary size of the growing text with the vocabulary size of the memoryless text, $N_{m}$, where we "forget" the words read in all previous texts and continue counting appearances of words that were initial in their text (regardless of appearances in previous texts). From $n_{m}$ and $N_{m}$ we then have two proxies for the word introduction rate, one for the growing text $\alpha_{m}=n_{m} / m$ and one for the memoryless text $A_{m}=N_{m} / m$. We may consider $\alpha_{m}$ to be the word introduction rate of the composite (which includes mixing effects) and $A_{m}$ to be the word introduction rate of the individual texts (excluding mixing effects).

There are many conceivable mechanisms that lead to a power-law decay in the rate of word introduction. To measure the severity of scaling breaks we do not need to know the true values of the word introduction rates but instead just their scalings. So, to determine the extent to which text mixing generates word introduction decay, we isolate the portion of the scaling that results from mixing by measuring $\alpha_{m} / A_{m}$, the portion of word introduction remaining after mixing texts. Note that since $n_{m} \leqslant N_{m}$, one has $\alpha_{m} \leqslant A_{m}$ and hence $\alpha_{m} / A_{m} \leqslant 1$ for all $m$. Hence, this normalized rate behaves as a nonconstant only when mixing ensues, and so any decay measured via $\alpha_{m} / A_{m}$ implies the presence, and is the direct consequence, of text mixing (see the example in Fig. 3 for an intuitive understanding of all text mixing quantities). Since $\alpha_{m} / A_{m}$ will be the only quantity used in the measurement of word introduction decay, we relax the notation and simply write $\alpha$ for $\alpha_{m} / A_{m}$ and $n$ for $n_{m}$ in what follows.

To test the effects of text mixing, we not only observe the word introduction rate $\alpha(n)$ but consider its ability to predict the scalings of rank-frequency distributions. To do this, we note that, by design, the data for $\alpha(n)$ are aligned with $f(r)$ —both have domain $\left\{1, \ldots, N_{\text {corp }}\right\}$ (where $N_{\text {corp }}$ is the vocabulary size of the corpus). Further, since the theory has $\gamma=\theta+\mu$, we may also observe that $\alpha(n) n^{-\theta}$ need only be normalized

$$
\hat{p}(n)=\frac{\alpha(n) n^{-\theta}}{C}, \quad \text { where } \quad C=\sum_{1}^{N_{\text {corp }}} \alpha(n) n^{-\theta},
$$

to produce a model for the normalized rank-frequency distribution $p(r)=f(r) / \sum_{1}^{N_{\text {corp }}} f(r)$. To determine a model's Zipf scaling, $\theta$, we scan the range $\{0.75,0.751, \ldots, 1.25\}$ and accept
Example: Consider the two excerpts from Charles Dickens' "A Tale of Two Cities", taken as texts:

$T_{1}$ : (it, was, the, best, of, times, it, was, the, worst, of, times), and

$T_{2}$ : (it, was, the, age, of, wisdom, it, was, the, age, of, foolishness)

Supposing we read $T_{1}$ first, the sequence of words is:

$$
\begin{aligned}
& \left(T_{1}, T_{2}\right): \text { (it, was, the, best, of, times, } \\
& \text { it, was, the, worst, of, times, } \\
& \text { it, was, the, age, of, wisdom, } \\
& \text { it, was, the, age, of, foolishness) }
\end{aligned}
$$

where initial (growing text) word appearances are presented in bold. The corresponding sequences of values, $m, n_{m}, N_{m}, \alpha_{m}, A_{m}$ and $\alpha_{m} / A_{m}$, are then

$$
\begin{aligned}
m: & (\mathbf{1}, \mathbf{2}, \mathbf{3}, \mathbf{4}, \mathbf{5}, \mathbf{6}, 7,8,9, \mathbf{1 0}, 11,12, \\
& 13,14,15, \mathbf{1 6}, 17, \mathbf{1 8}, 19,20,21,22,23, \mathbf{2 4}) \\
n_{m}: & (\mathbf{1}, \mathbf{2}, \mathbf{3}, \mathbf{4}, \mathbf{5}, \mathbf{6}, 6,6,6, \mathbf{7}, 7,7, \\
& 7,7,7, \mathbf{8}, 8, \mathbf{9}, 9,9,9,9,9, \mathbf{1 0}) \\
N_{m}: & (\mathbf{1}, \mathbf{2}, \mathbf{3}, \mathbf{4}, \mathbf{5}, \mathbf{6}, 6,6,6, \mathbf{7}, 7,7, \\
& 8,9,10, \mathbf{1 1}, 12, \mathbf{1 3}, 13,13,13,13,13, \mathbf{1 4}) \\
\alpha_{m}: & \left(\mathbf{1}, \mathbf{1}, \mathbf{1}, \mathbf{1}, \mathbf{1}, \mathbf{1}, \frac{6}{7}, \frac{6}{8}, \frac{6}{9}, \frac{\mathbf{7}}{\mathbf{1 0}}, \frac{7}{11}, \frac{7}{12},\right. \\
& \left.\frac{7}{13}, \frac{7}{14}, \frac{7}{15}, \frac{\mathbf{8}}{\mathbf{1 6}}, \frac{8}{17}, \frac{\mathbf{9}}{\mathbf{1 8}}, \frac{9}{19}, \frac{9}{20}, \frac{9}{21}, \frac{9}{22}, \frac{9}{23}, \frac{\mathbf{1 0}}{\mathbf{2 4}}\right) \\
A_{m}: & \left(\mathbf{1}, \mathbf{1}, \mathbf{1}, \mathbf{1}, \mathbf{1}, \mathbf{1}, \frac{6}{7}, \frac{6}{8}, \frac{6}{9}, \frac{\mathbf{7}}{\mathbf{1 0}}, \frac{7}{11}, \frac{7}{12},\right. \\
& \left.\frac{8}{13}, \frac{9}{14}, \frac{10}{15}, \frac{\mathbf{1 1}}{\mathbf{1 6}}, \frac{12}{17}, \frac{\mathbf{1 3}}{\mathbf{1 8}}, \frac{13}{19}, \frac{13}{20}, \frac{13}{21}, \frac{13}{22}, \frac{13}{23}, \frac{\mathbf{1 4}}{\mathbf{2 4}}\right) \\
\frac{\alpha_{m}}{A_{m}}: & (\mathbf{1}, \mathbf{1}, \mathbf{1}, \mathbf{1}, \mathbf{1}, \mathbf{1}, 1,1,1, \mathbf{1}, 1,1, \\
& \left.\frac{7}{8}, \frac{7}{9}, \frac{7}{10}, \frac{\mathbf{8}}{\mathbf{1 1}}, \frac{8}{12}, \frac{\mathbf{9}}{\mathbf{1 3}}, \frac{9}{13}, \frac{9}{13}, \frac{9}{13}, \frac{9}{13}, \frac{9}{13}, \frac{\mathbf{1 0}}{\mathbf{1 4}}\right) .
\end{aligned}
$$

FIG. 3. A concrete example of the text mixing effect, where we consider two passages $\left(T_{1}\right.$ and $\left.T_{2}\right)$ as separate texts that are then mixed. The similarity of word use between these excerpts provides an excellent example for understanding the differences between the growing text, where we count new word appearances $\left(n_{m}\right)$ with the awareness of previous texts, and the memoryless text, where we count word appearances $\left(N_{m}\right)$ as new with each initial appearance in each text. Note that both $\alpha_{m}$ and $A_{m}$ are simply the quotients of $n_{m}$ and $N_{m}$ with $m$ (respectively) and that their quotient $\left(\alpha_{m} / A_{m}\right)$ is equivalent to $n / N$ and is not equal to 1 only when texts are mixed.

the $\theta$ for which $\hat{p}$ minimizes the sum of squares error

$$
\sum_{1}^{N_{\text {corp }}}\left[\log _{10} p(r)-\log _{10} \hat{p}(r)\right]^{2}
$$

over as many as 10000 log-spaced ranks.

\section{MATERIALS AND METHODS}

In our experiments we worked with a subset of the eBooks [13] collection. We collected those texts which were annotated sufficiently well to allow for the removal of metadata as well as for the parsing of authorship, title, and language. 
TABLE I. Table of information concerning the data used from the eBooks database. For each language we record the number of books $\left(N_{\text {books }}\right)$; the number of characters $\left(N_{\text {char }}\right)$, which we take to be the number of letters $[14,15]$ (including diacritics and ligatures); the minimum text size $\left(N_{\min }\right)$; the maximum text size $\left(N_{\max }\right)$; and the total corpus size $\left(N_{\text {corp }}\right)$. For reference, we additionally record the regressed point of scaling break, $b$.

\begin{tabular}{cccccccc}
\hline \hline & $N_{\text {books }}$ & $N_{\text {char }}$ & $N_{\text {min }}$ & $N_{\text {ave }}$ & $b$ & $N_{\text {max }}$ & $N_{\text {corp }}$ \\
\hline en & 19793 & 46 & 5 & 5899.3 & 5849 & 219990 & 2836900 \\
fr & 1360 & 44 & 395 & 8300.7 & 17715 & 26171 & 528314 \\
fi & 505 & 31 & 1144 & 8872.6 & 7761 & 31623 & 811742 \\
nl & 434 & 48 & 133 & 6747.1 & 6098 & 82246 & 443816 \\
pt & 375 & 38 & 203 & 4675.8 & 10363 & 17818 & 246497 \\
de & 327 & 30 & 153 & 7554.9 & 7259 & 113089 & 477274 \\
es & 223 & 34 & 406 & 8735.1 & 15079 & 29452 & 237874 \\
it & 194 & 29 & 1083 & 9388.7 & 13954 & 29445 & 258509 \\
sv & 56 & 34 & 1389 & 7499.8 & 5315 & 18726 & 123806 \\
el & 42 & 35 & 2047 & 6414.7 & 7613 & 17774 & 110940 \\
\hline \hline
\end{tabular}

Altogether, this resulted in the inclusion of 23309 books from across 10 languages (broken down in Table I).

To idealize texts as discussed in Fig. 1, we note that a resultant rank-frequency distribution from a pure Simon model of constant word introduction rate, $\alpha_{0}$, will scale with Zipf exponent $\theta=1-\alpha_{0}$ such that $N / M \rightarrow \alpha_{0}$ as the text grows. Therefore, for an observed text of size $N$ and volume $M$, we define the idealized Zipf-Simon exponent as $\theta_{0}=1-N / M$, and apply $\theta_{0}$ to the collection of ranks, $r=1, \ldots, N$, as

$$
f_{\text {ideal }}(r)=\left\lfloor\left(\frac{r}{N}\right)^{-\theta_{0}}+\frac{1}{2}\right\rfloor,
$$

while preserving their word labels from the empirical data.

For all of the rank-frequency distributions analyzed, we regress over as many as 10000 log-spaced ranks (taken over the range $r=1, \ldots, N$ ) to determine estimates for $\theta, b$, and $\gamma$. This estimation is done by applying a two-line least-squares regression, constrained by intersection at the point of scaling break. Given data points $(x, y)$ and a point of break, $x_{b}$, we solve for the model

$$
\hat{y}=\left\{\begin{array}{ll}
\beta_{1}+\beta_{2} x, & : x \leqslant x_{b} \\
\beta_{3}+\beta_{4} x, & : x>x_{b}
\end{array},\right.
$$

constrained by $\beta_{1}+\beta_{2} x_{b}=\beta_{3}+\beta_{4} x_{b}$, through standard minimization of the sum of squares error. We compute this regression for $1000 \log$-spaced points, $x_{b}$, across the middle $20-80 \%$ of the $\log r$ domain. For a given distribution we then perform these 1000 regressions and accept the value $b$ for which we have observed the smallest sum of squared errors.

To understand our text mixing results we must note that there is measurement error for both $b$ and $N_{\text {avg. }}$. As a regressed quantity, this may be expected for $b$, but for $N_{\text {avg }}$, the existence of measurement error is less obvious and generally results from poor corpus composition. The main effect stems from the fact that many texts in the eBooks data set are internally mixed. The longitudinal compendia of individual authors and genres are the most intuitive and abundant examples of internally mixed texts, and the most extreme cases are generally reference texts, e.g., dictionaries, encyclopedias, and textbooks (see Fig. 2).

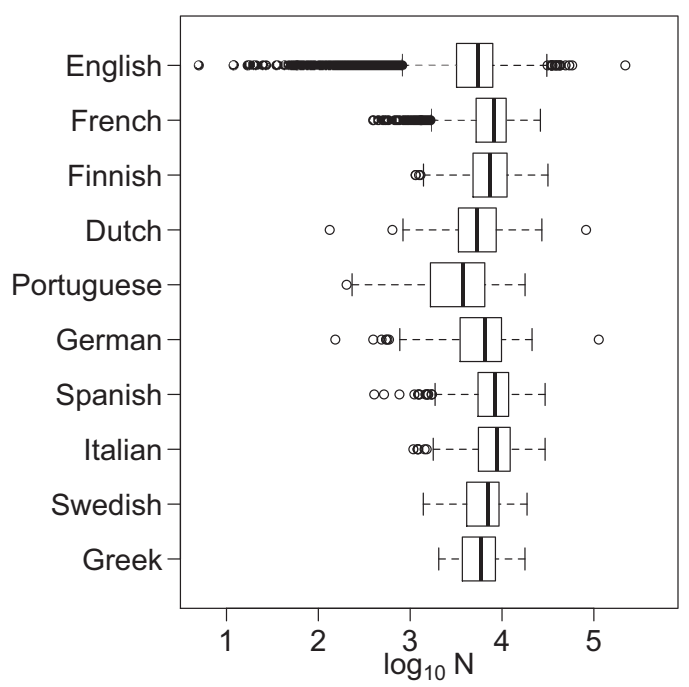

FIG. 4. Box plots of the base-10 logarithm vocabulary sizes of the texts contained in the 10 eBooks corpora studied. Center bars indicate means and whiskers extend to most extremal values up to 1.5 times the interquartile-range length, whereupon more extremal values are plotted as points designated "outliers."

The major point is that when a compendium is not refined, but taken as an individual text in a corpus, the calculation of $N_{\text {avg }}$ considers only a single book of large size (wrongly), instead of many books of smaller size (correctly). Within the English data set we have found that the large- $N$ texts are generally of this variety and dominate the $10^{\text {th }}$ decile. Reading down the top ten $N$-ranking texts makes this abundantly clear:

1. Webster's Unabridged Dictionary

2. Diccionario Ingles-Español-Tagalog

3. The Complete Project Gutenberg Works of George Meredith

4. The Anatomy of Melancholy

5. A Concise Dictionary of Middle English

6. A Pocket Dictionary

7. The Nuttall Encyclopaedia

8. The Complete PG Works of Oliver Wendell Holmes, Sr.

9. The Complete Historical Romances of Georg Ebers

10. The Complete Project Gutenberg Works of Galsworthy Note here that among these compendia and reference texts lies a two way (Spanish-English) dictionary whose placement in the top 10 likely results from dual word forms (English and Spanish translations) of the majority of words that it possesses. We have explored the impact of these under-refined and illformed texts in detail in Fig. 2, where we have found a clear association of $b$ with $N_{\text {avg }}$ along the line $b=N_{\text {avg }}$ that breaks down in the larger deciles, where these strange texts occur.

We also note that $N_{\text {avg }}$ is subject to measurement error from over-refined texts as well, most notably in the Portuguese data set, which has the smallest average text size, while having the fifth largest number of books (see Table I and Fig. 4). There we note that Portuguese presents the most significant deviation between $N_{\text {avg }}$ and $b$ ( $b$ is notably more than $120 \%$ larger than $N_{\text {avg }}$ ), and, moreover, that this deviation is in the expected direction, i.e., $N_{\text {avg }} \ll b$. Note also that this observation is in agreement with those other languages that have $N_{\text {avg }} \ll b$ in 
Table I (specifically Italian, Spanish, and French), where in Fig. 4 we see that having many low- $N$ outliers with no high- $N$ outliers biases the corpuswide measurement of $N_{\text {avg }}$.

To estimate $\mu$ we perform common least-squares linear regression on the log-transformed data over the region $\left[N_{\text {avg }}, N_{\text {corp }}\right]$, since $N_{\text {avg }}$ is generally the point at which mixingderived decay becomes clear.

Computation of $\alpha(n)$ involves running many realizations of the text mixing procedure, randomizing the order in which the texts are read. To ensure that our measurements are accurate, we adhere to a heuristic - that the number of text mixing runs be no less than $10 \times N_{\text {books }}$ for the given corpus. The final values used is in our experiments are computed as averages of the $\alpha_{m} / A_{m}$ from the more than $10 \times N_{\text {books }}$ runs. However, we note that $\alpha_{m} / A_{m}=n_{m} / N_{m}$, where $n_{m}$ ranges with rank: $n_{m}=1,2,3, \ldots, N_{\text {corp }}$. So the only quantities that vary across runs that are necessary to compute $\alpha(n)$ are the $N_{m}$. Hence we take the average as $\alpha\left(n_{m}\right)=n_{m} /\left\langle N_{m}\right\rangle$, which is in fact the harmonic mean of the $\alpha\left(n_{m}\right)$ (the truest mean for rates).

In our investigation of the different divisions of the internally mixed corpus, The Complete Historical Romances of Georg Ebers (Fig. 7), we have shown how important it is to have meaningfully defined texts to be able to produce an accurate text mixing model for a corpus. An important component of this exhibition is the extremal refinement, where each word is treated individually as a separate text (a highly nonrealistic scenario). To conduct a text mixing experiment for such a refinement can be quite computationally taxing, as this requires taking permutations of the word orders of the entire corpus. Since this process is entirely independent of the original word orderings from the corpus, it may be computed directly from the rank-frequency distribution via expected gap sizes. In particular, we wish to determine the average number of previously seen words appearing between the $n^{\text {th }}$ and $n+1^{\text {st }}$ "new" words, given all permutations of the corpus words. Denoting this number by $\bar{M}_{n}$, we note that the average word introduction rate over this range is easily found as $\alpha_{n}=1 / \bar{M}_{n}$. We then define $i_{n}$ as the total number of previously observed words that were not yet counted by the time the $n^{\text {th }}$ new word was observed and define $j_{n}$ to be the total number (out of all corpus words) that were not yet counted by the time the $n^{\text {th }}$ new word was first observed (including those word types that were not yet observed). Then, if $P_{n}(M)$ is the probability that

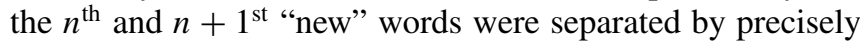
$M$ previously seen words,

$$
\begin{aligned}
\bar{M}_{n} & =\sum_{M=0}^{i_{n}} M P_{n}(M) \\
& =\sum_{M=0}^{i_{n}} M \frac{j_{n}-i_{n}}{j_{n}-M} \prod_{k=0}^{M-1} \frac{i_{n}-k}{j_{n}-k},
\end{aligned}
$$

where, in the last expression, the product is the probability of seeing $M$ consecutive previously observed words, with the first factor being the probability that the "new" word is seen as the $M+1^{\text {st }}$. These expressions for the $\bar{M}_{n}$ are iteratively computable and, in addition, since the sums appear (empirically) to converge quickly, we find that it suffices to take their first 1000 terms for added computational efficiency.

\section{RESULTS AND DISCUSSION}

To understand our results we define $N_{\min }, N_{\text {avg }}$, and $N_{\max }$ as the minimum, average, and maximum text sizes (by numbers of unique words), respectively (see Table I). These three values define four text mixing regimes:

$$
\begin{aligned}
n<N_{\min } ; \text { Zipf-Simon (no mixing) } \\
N_{\min } \leqslant n \leqslant N_{\text {avg }} ; \text { initial (minimal mixing) } \\
N_{\text {avg }} \leqslant n \leqslant N_{\text {max }} ; \text { crossover (partial mixing) } \\
n>N_{\text {max }} ; \text { terminal (full mixing). }
\end{aligned}
$$

In the Zipf-Simon regime we expect the result of an unperturbed Simon model, but because mixing is also minimal over the initial regime, we expect the behavior over the first two regimes to be more or less consistent. Once in the crossover regime, words will on average have appeared under the effects of text mixing and so there is the expectation that $N_{\text {avg }}$ will mark the macroscopically observable change in behavior or scaling break of the rank-frequency distribution, i.e., we expect $b \approx N_{\text {avg. }}$. Plotting the two against one another, we see that this relationship holds across sample corpora from the well-behaved deciles of the English distribution of text sizes (see Fig. 2) and breaks down in the presence of ill-formed texts. Finally, over the terminal regime, all words will appear in the

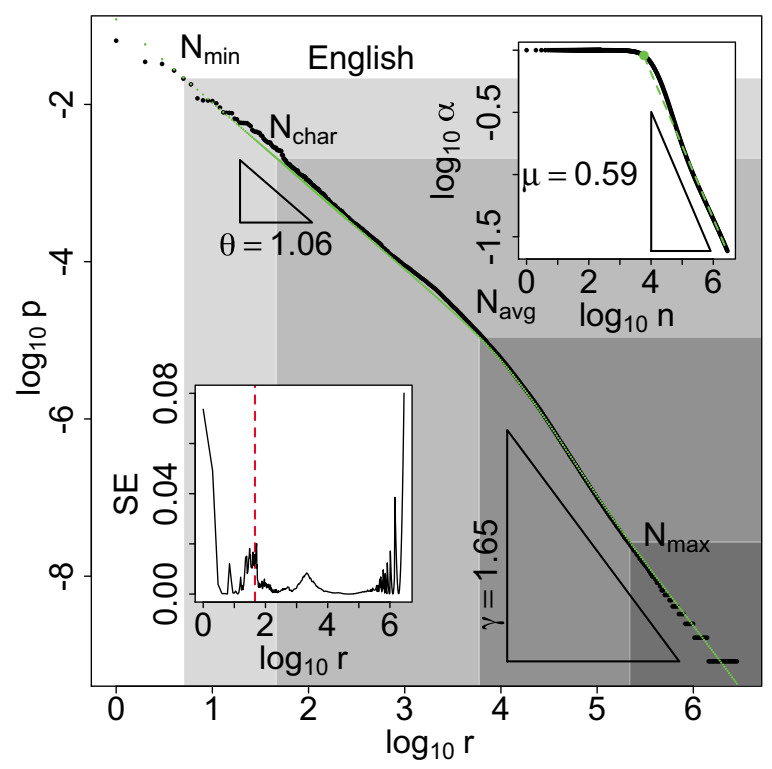

FIG. 5. (Color online) The results of text mixing experiments for the largest, English corpus from the eBooks collection. The main axes show the empirical, normalized rank-frequency distribution (black points), $p$, and the model determined by text mixing [green (gray) points], $\hat{p}$. The measured lower and upper exponents, $\gamma$ and $\theta$, are depicted in the lower right and upper left, respectively, with triangles indicating the measured slopes. We also present gray boxes in the main axes to highlight the different mixing regimes, marked by $N_{\text {char }}$, $N_{\text {min }}, N_{\text {avg }}$, and $N_{\text {max }}$ (see Sec. IV and Table I for complete descriptions of these quantities). The lower left inset shows the pointwise squared error $[p(r)-\hat{p}(r)]^{2}$, whose sum is minimized in the transformation of $\alpha$ into $\hat{p}$. The upper right inset shows the untransformed rate of word introduction, $\alpha$ (black points), and the decay exponent $\mu$, which is depicted by the regressed slope [green (gray) dashed line]. 

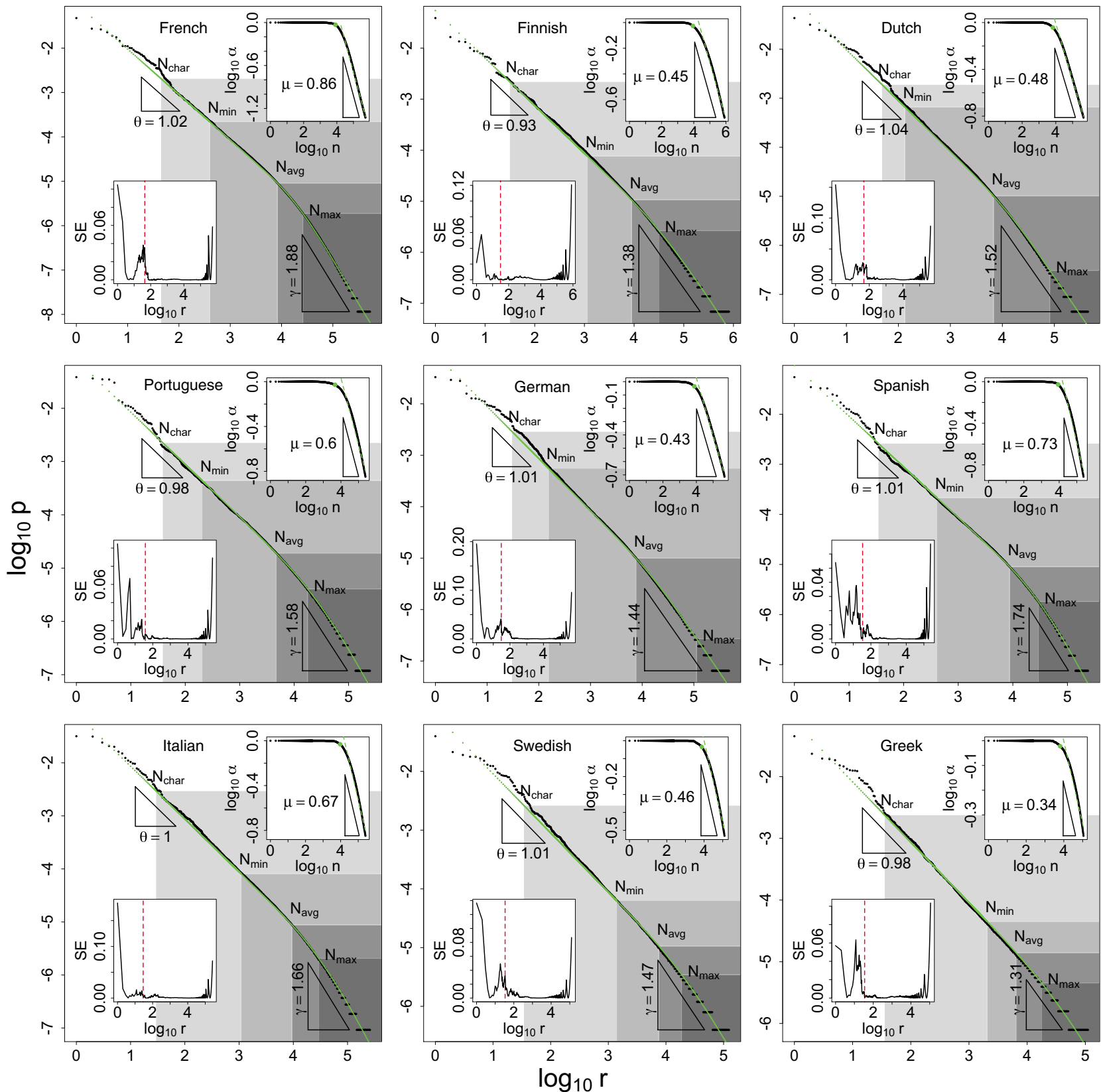

FIG. 6. (Color online) The results of text mixing experiments for the nine smaller corpora analyzed. All insets, color coding, and labels are consistent with those from the larger, English presentation in Fig. 5, whose caption possesses full descriptions of all axes and plotted data.

presence of mixing, and so this regime exhibits the stabilized second scaling, characterized by the decay parameter $\mu$.

Our main results from text mixing, comparing the textmixing-derived model, $\hat{p}$, with the normalized empirical rankfrequency data, $p$, may be found for the English data set in Fig. 5 and for the nine other languages studied in Fig. 6. For all 10 languages we observe that the models defined by text mixing, $\hat{p}$, produce excellent predictions of the rank-frequency distributions (main axes in Figs. 5 and 6), which is made quite clear by plotting pointwise squared error (lower-left insets in Figs. 5 and 6). For each corpus we see a broad range of ranks beginning not far before $10^{2}$ and extending into the second scaling where the error is quite low (disregarding the effect of the finite-size plateaux).
We also perform text mixing analysis at different scales for a single, large, and internally mixed text from the English data set, The Complete Historical Romances of Georg Ebers. It is important to note before interpreting these results that the text itself is a compendium, combining series that were each written by the author over the course of more than 30 years, writing and publishing volumes independently. With this in mind, the text offers an important example for text mixing that helps us to understand several important details: first, that not all texts are well formed-an individual text such as this may in and of itself present a scaling break that has resulted from text mixing; second, that the scaling break of a single, large text may be understood through text mixing analysis. This second point is more difficult to observe, as it requires an appropriate 
refinement of the internally mixed text, i.e., one must be able to break the mixed text into appropriately independent subtexts. From our example in Fig. 7, we can see that the division of the text into a corpus of 28 series (left panel) renders a text mixing model for the empirical data with much higher error than a division into a corpus of 143 volumes (center panel, a refinement of the series' division). We also present text mixing results from the extremal refinement, where each individual word is treated as a text (right panel, see Sec. IV for more information on the extremal refinement), which shows that a text can be over-refined to produce a poor text mixing model.

It is worth noting from our results that the parameter, $\theta$, is frequently measured to lie outside the Simon-productive range, $(0,1)$. Therefore, we are left to conclude that, individually, many texts are subject to internally derived decay in word introduction rates (as is exemplified by the Ebers text in Fig. 7), i.e., the underlying rank-frequency distributions are not of pure Zipf-Simon form (as we suggest in other work [8]) but, instead, subject to internal mixing. Though we do not exhaustively investigate the occurrence of internally derived decay in the rates of word introduction across the eBooks data set, it seems quite possible that all of the texts parsed are subject to some internal mixing effects, whether from nonoriginal annotation by the Project Gutenberg e-Text editors or just the mixing of differing components (e.g., chapters, series, volumes, prologues, etc.). This of course would require that these mixing effects be of low impact in the cases generally considered strong examples of Zipf's law.

We also note a strange behavior (which is captured by the text mixing model) in the English data set. There we have found a relatively shallow lower scaling $(\gamma \approx 1.65)$, but notice that it appears to be one of possibly two lower scalings. For English, the crossover regime exhibits a consistently steeper scaling that dies away in the terminal regime. Though we have no certain explanation for this behavior, part of what makes the English collection differ so much from the others is the sheer number of texts (see Table I). However, upon looking closer at the distribution of English text sizes, we also notice that the collection possess some extremely large- $N$ outliers. In the largest text (which has nearly an order of magnitude more words than any other text), approximately $1 / 10$ of all words are represented (of nearly 20000 books), which must have a profound impact on the combined rank-frequency distribution and hence lower scaling. Further, this large- $N$ hypothesis is supported by our preliminary investigation (see Fig. 2) where we observed that those (large) texts in the 10th decile not only generated scaling break points that went against the $b=N_{\mathrm{avg}}$ correspondence but also generated relatively shallow lower scalings, against the trend of steepening with increasing decile. English is also well known for its willingness to adopt foreign words, which may lead to an increased rate of appearance of low-count loan words. Regardless of the reasons for this difference with English, we find that text mixing captures the shape of both lower scaling regimes, and so both are well explained by the text mixing model.

We also take time to make note of and discuss another anomalous behavior of the rank-frequency distributions investigated. Upon viewing a rank-frequency distribution for Zipf's law, one generally finds a "wobble" of the frequency data around Zipf's scaling (regardless of the existence of a scaling break). We refer to the termination of this "wobble" as the point of stabilization of the Zipf-Simon regime. Looking at the empirical data from the 10 languages, we see that this stabilization point generally appears early on in the Zipf-Simon regime and generally not before the first $10^{2}$ ranks. Though we have no definitive explanation for the existence of this anomaly, we note upon looking at the pointwise-squared errors that the stabilization point frequently occurs near each language's number of characters, $N_{\text {char }}$ (depicted as a red dotted vertical line in each of the lower left insets of Figs. 5 and 6 and the center panel of Fig. 7). Whether the numbers of characters spawned in the generation of primordial, characterbased languages still influence the shapes of rank-frequency distributions of descendant languages today, we cannot say
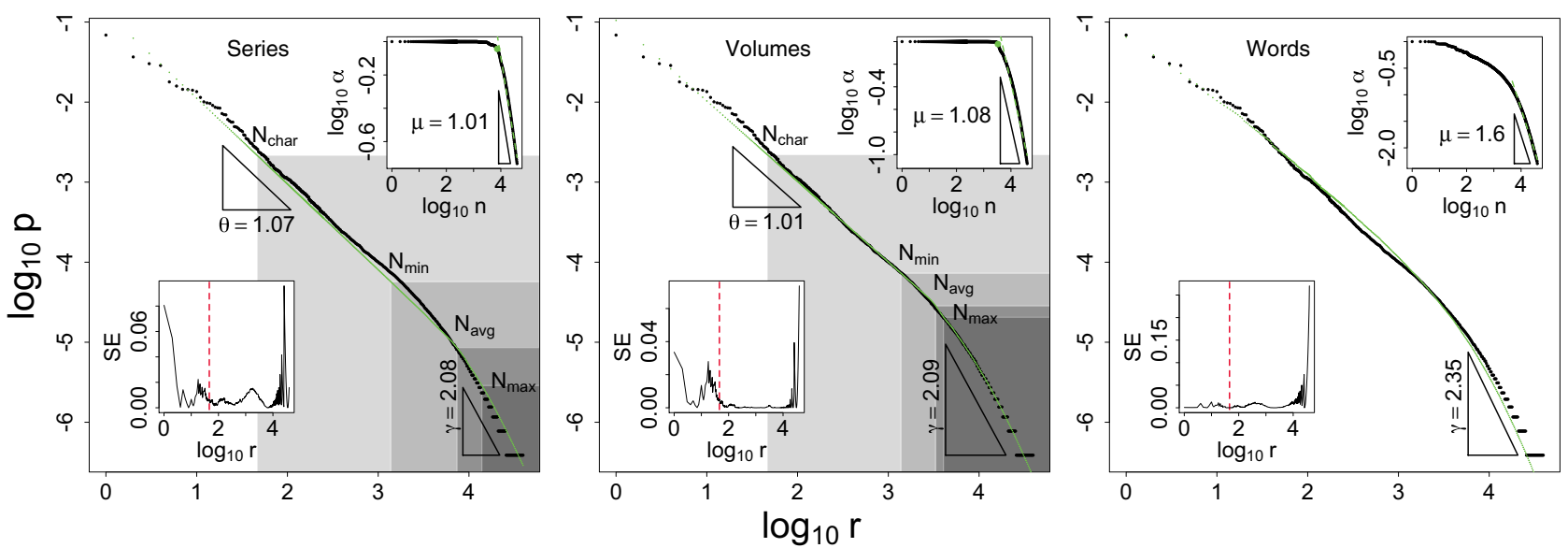

FIG. 7. (Color online) Text mixing results for a single-author corpus. Here $\alpha$ was measured for differing refinements of the Egyptological fiction compendium-text The Complete Historical Romances of Georg Ebers into subtexts. All insets, color coding, and labels are consistent with those from the English presentation in Fig. 5, whose caption possesses full descriptions of all axes and plotted data. Left: Each series is considered a separate text. Middle: Each volume of each series is considered a separate text. Right: Each word (the extremal refinement, see Sec. IV) in the compendium is considered a separate text. Note that in the upper right insets, $\alpha$ decreases overall with each refinement (as by definition it must) and that there appears to be an optimal refinement for producing a text mixing model, likely close to the scale of volumes. 
for sure. However, this anomalous regime appears consistently across languages and may potentially be of consistent shape across the corpora of a language. If so, we might view such anomalies as universal properties of languages and so highlight them in the hopes of opening a broader discussion.

In light of the results presented, we take time to consider the validity of the core language hypothesis. We have seen significant variation in both the location and severity of scaling breaks both across and within languages. Upon sampling the English corpus by deciles, we have observed that the regressed point of scaling break, $b$, is not stationary (see Fig. 2). We take this as indication of the lack of validity of and language-universal core-noncore hypothesis, as a core should exhibit a strong consistency of size. Moreover, languages closely related via a common, recent ancestor should likewise exhibit this consistency, but notably two of the languages most closely related in the study, Spanish and Portuguese, present a large difference in $b$ (10363 for Spanish, and 15079 for Portuguese-see Table I). Both of these results seem to indicate that scaling breaks in rank-frequency distributions are likely consequences of text and corpus composition. Hence, it may then be more reasonable to consider a language core as a collection of words necessary for basic description but not overlapping in use or meaning. However, such a core lexicon would need to be determined by native practitioners and not necessarily be an observable property of rank-frequency distributions. Alternatively, one could consider a corpus core by its collection of words common to its texts. However, such a "common core" would be entirely dependent on the composition of the corpus and hence not a universal property of a language proper.
[1] G. K. Zipf, The Psycho-Biology of Language (Houghton-Mifflin, New York, 1935).

[2] G. K. Zipf, Human Behaviour and the Principle of Least-Effort (Addison-Wesley, Boston, 1949).

[3] R. Ferrer-i-Cancho and R. V. Solé, J. Quant. Linguist. 8, 165 (2001).

[4] M. A. Montemurro, Physica A 300, 567 (2001).

[5] M. Gerlach and E. G. Altmann, Phys. Rev. X 3, 021006 (2013).

[6] J. Kwapien, S. Drozdz, and A. Orczyk, Acta. Phys. Pol. A 117, 716 (2010).

[7] A. M. Petersen, J. N. Tenenbaum, S. Havlin, H. E. Stanley, and M. Perc, Sci. Rep. 2, 943 (2012).
[8] J. R. Williams, P. R. Lessard, S. Desu, E. Clark, J. P. Bagrow, C. M. Danforth, and P. S. Dodds, arXiv:1406.5181 (2014).

[9] H. A. Simon, Biometrika 42, 425 (1955).

[10] G. U. Yule, Phil. Trans. B 213, 21 (1924).

[11] A. L. Barabási and R. Albert, Science 286, 509 (1999).

[12] P. L. Krapivsky and S. Redner, Phys. Rev. E 63, 066123 (2001).

[13] http://www.gutenberg.org, accessed July 1, 2014.

[14] https://en.wikipedia.org/wiki/Latin_alphabets, accessed November 1, 2014.

[15] https://en.wikipedia.org/wiki/Greek_alphabet, accessed November 1, 2014. 